\title{
Dysregulation of zinc/lipid metabolism-associated genes in the rat hippocampus and cerebral cortex in early adulthood following recurrent neonatal seizures
}

\author{
ZHEN-HONG LI ${ }^{1,2}$, LI-LI LI ${ }^{1}$, MEI-FANG JIN ${ }^{1}$, XU-QIN CHEN ${ }^{1}$, QI SUN ${ }^{1}$ and HONG NI ${ }^{1}$ \\ ${ }^{1}$ Neurology Laboratory, Institute of Pediatrics, Children's Hospital of Soochow University, \\ Suzhou, Jiangsu 215003; ${ }^{2}$ Department of Pediatrics, Ganzhou City People's Hospital/Ganzhou \\ Hospital Affiliated to Nanchang University, Ganzhou, Jiangxi 341000, P.R. China
}

Received August 22, 2016; Accepted June 6, 2017

DOI: $10.3892 / \mathrm{mmr} .2017 .7160$

\begin{abstract}
Although it has been established that recurrent or prolonged clinical seizures during infancy may cause lifelong brain damage, the underlying molecular mechanism is still not well elucidated. The present study, to the best of our knowledge, is the first to investigate the expression of twenty zinc (Zn)/lipid metabolism-associated genes in the hippocampus and cerebral cortex of rats following recurrent neonatal seizures. In the current study, 6-day-old Sprague-Dawley rats were randomly divided into control (CONT) and recurrent neonatal seizure (RS) groups. On postnatal day 35 (P35), mossy fiber sprouting and gene expression were assessed by Timm staining and reverse transcription-quantitative polymerase chain reaction, respectively. Of the twenty genes investigated, seven were significantly downregulated, while four were significantly upregulated in the RS group compared with CONT rats, which was observed in the hippocampus but not in the cerebral cortex. Meanwhile, aberrant mossy fiber sprouting was observed in the supragranular region of the dentate gyrus and Cornu Ammonis 3 subfield of the hippocampus in the RS group. In addition, linear correlation analysis identified significant associations between the expression of certain genes in the hippocampus, which accounted for $40 \%$ of the total fifty-five gene pairs among the eleven regulated genes. However, only eight gene pairs in the cerebral cortex exhibited significant positive associations, which accounted for $14.5 \%$ of the total. The results of the present study indicated the importance of hippocampal $\mathrm{Zn} /$ lipid metabolism-associated genes in recurrent neonatal seizure-induced aberrant mossy fiber
\end{abstract}

Correspondence to: Professor Hong Ni, Neurology Laboratory, Institute of Pediatrics, Children's Hospital of Soochow University, 303 Jingde Road, Suzhou, Jiangsu 215003, P.R. China

E-mail: nyr2000@yeah.net

Key words: zinc transporter, lipid metabolism, hippocampus, mossy fiber sprouting, neonatal seizure sprouting, which may aid the identification of novel potential targets during epileptogenesis.

\section{Introduction}

Neonatal seizures represent a heterogeneous group of clinical pediatric epileptology. While prolonged or recurrent developmental seizures may induce long-term neurobehavioral, cognitive and pathological sequelae $(1,2)$, neonatal hypocalcemic or hypoglycemic seizures in the first week of life, on the other hand, may be benign. Furthermore, neonatal seizures may be refractory to antiepileptic drugs such as phenobarbital, which has exhibited long-term cognitive and intellectual impairment for febrile seizures (3). Therefore, an improved understanding of the molecular mechanisms of altered brain maturation and function following neonatal seizures is required before age-appropriate treatments can be optimized.

The hippocampus is more vulnerable to epileptic insults compared with the cerebral cortex. Hippocampal sclerosis is a neuropathological condition that may be observed in mesial temporal lobe epilepsy (MTLE) and other epilepsy syndromes, which may be identified in surgery or post-mortem (4). Permanent hippocampal changes that develop over weeks include circuit organization, which may be identified by aberrant mossy fiber sprouting and functional properties in adult hippocampal circuitry, and may influence epileptogenesis (5). In fact, aberrant sprouting of hippocampal mossy fibers has been recognized as a pathological hallmark of MTLE (6).

Recently, abnormal zinc ( $\mathrm{Zn}$ ) metabolism has been reported to be associated with hippocampal mossy fiber sprouting. Zn deficiency or excess Zn contributes to impaired behavior and brain developmental disorders (7). Brain $\mathrm{Zn}$ is abundantly distributed in the Zn-rich mossy fiber pathway in the hippocampus, which is important in maintaining a balance between excitatory and inhibitory systems, particularly in the pathological process of epileptogenesis following an initial brain-damaging insult (8). Zn transporters have an important role in the deleterious changes of axon sprouting. We previously reported long-term downregulated expression of $\mathrm{Zn}$ transporter (ZnT)-1 and upregulated expression of ZnT-3 in the rat hippocampus following volatile flurothyl-induced 
neonatal or penicillin-induced developmental seizures, which is in parallel with increased regenerative hippocampal mossy fiber sprouting (9-11). However, we did not determine the expression pattern of other $\mathrm{Zn}$ transporters.

We previously reported altered expression of various lipid metabolism-associated genes in the hippocampus following neonatal seizures, including apolipoprotein $\mathrm{J}$, apolipoprotein E, acetyl-CoA acetyltransferase 1 and plasticity-related genes (PRGs) (12). Using an identical neonatal seizure model, we recently demonstrated significant upregulated expression of ZnT-3, metallothionein-3 (MT-3) and lipid metabolism-associated apolipoprotein E, clusterin and acetyl-CoA acetyltransferase 1 in the hippocampus, and also reported that a ketogenic diet blocked these effects (13). Therefore, we hypothesized that an interaction between $\mathrm{Zn}$ and lipid metabolism signals may exist in the experimental neonatal seizure model.

The aim of the present study was to investigate the long-term expression of $\mathrm{Zn}$ transporter and lipid metabolism-associated genes following neonatal seizures. Unlike in our previous studies, the present study evaluated the gene expression in early adulthood on postnatal day 35 (P35; 21 days after the last neonatal seizure). In addition, the current study compared the expression of $\mathrm{Zn}$ transporters with lipid metabolism-associated gene expression. Furthermore, the hippocampus and cerebral cortex were analyzed as it has been reported that status epilepticus in the immature rat induced neuronal degeneration in multiple regions outside the hippocampus (14), and also because pediatric cortical dysplasia may also have key roles in epileptogenesis (15).

\section{Materials and methods}

Animal preparation. Sprague-Dawley rats with balanced sex distribution (weight, 11-13 g; supplied by Shanghai SLAC Laboratory Animal Co., Ltd., Shanghai, China) were treated in accordance with the guidelines set by the National Institutes of Health (Bethesda, MD, USA) for the humane treatment of animals. Ethical approval was obtained from the Medical Ethics Committee of the Children's Hospital of Soochow University. Rats $(n=24)$ at postnatal day 6 (P6) were assigned to the following two groups: Recurrent-seizures (RS) group $(n=12)$; and control group $(n=12)$. The procedure of seizure induction has been described in detail previously (16). In brief, neonatal rats were placed into a transparent plastic airtight box and liquid volatile flurothyl (bis-2, 2, 2-triflurothyl ether; Sigma-Aldrich; Merck KGaA, Darmstadt, Germany) was delivered through a syringe. The experimental rats were subjected to forty-five induced seizures during nine consecutive days, between P6 and P14. Rats had five seizures per day for nine consecutive days, with a minimum of $30 \mathrm{~min}$ between seizures. Control rats were placed into the container for an equal amount of time without exposure to flurothyl. The litters were weaned on postnatal day 21 , and following this age they were housed in an environment-controlled room which was away from bright light and noise (temperature $22 \pm 1^{\circ} \mathrm{C}$; humidity $70 \pm 5 \%$ ) under a $12 / 12 \mathrm{~h}$ standard light-dark cycle and had access to standard laboratory food and water ad libitum. Animals were sacrificed by decapitation at P35 for Timm staining and reverse transcription-quantitative polymerase chain reaction (RT-qPCR) experiments.

Timm staining. Six rats from each group were anesthetized intraperitoneally with $4 \%$ chloral hydrate $(1 \mathrm{ml} / 100 \mathrm{~g}$; Sangon Biotech Co., Ltd., Shanghai, China). The method has been described in detail previously (9).

$R T-q P C R$. This method has been described in detail previously (10). The primers and Taqman probes of the twenty genes were designed based on GenBank-published sequences with the software Primer Express 2.0 (Applied Biosystems; Thermo Fisher Scientific, Inc., Waltham, MA, USA), the sequences of which are listed in Table I. Thermocycling conditions for qPCR (Applied Biosystems 7500 Real-Time PCR System; Thermo Fisher Scientific, Inc.): 40 cycles $\left(95^{\circ} \mathrm{C}\right.$, $15 \mathrm{sec}$ for denaturation; $60^{\circ} \mathrm{C}, 1 \mathrm{~min}$ for annealing/extension). The experiment was repeated two times. The $2^{-\Delta \Delta \mathrm{Cq}}$ method of relative quantification was used to determine the fold change in expression. Initially, the qPCR threshold cycle $(\mathrm{Cq})$ of the target mRNAs and the internal control $\beta$-actin was determined. Subsequently, the ratios of target genes: $\beta$-actin were calculated as follows: Target gene: $\beta$-actin $=2^{\mathrm{Cq}(\text { target })-\mathrm{Cq}(\beta \text {-actin })}$ $(\Delta \mathrm{Cq}=\mathrm{Cq}$ target-Cq $\beta$-actin) (17). The fold change in expression was then obtained.

Statistical analysis. Statistical analysis of the changes in gene expression levels $\left(2^{-\Delta \Delta \mathrm{Cq}}\right)$ was performed using one-way analysis of variance followed by Dunnett's test. Linear correlations were analyzed by Pearson correlation analysis. SAS 8.0 statistical software (SAS Institute, Cary, NC, USA) was used to perform statistical analysis. Data are presented as the mean + standard deviation. $\mathrm{P}<0.05$ was considered to indicate a statistically significant difference.

\section{Results}

Timm staining. No obvious mossy fiber terminals in the control hippocampus (Fig. 1A) were observed. However, prominent aggregation of mossy fiber terminals was observed in the supragranular region of the dentate gyrus and CA3 subfield in the RS group (Fig. 1B).

RT-qPCR analysis. RT-qPCR was performed to investigate changes in the relative gene expression of $\mathrm{Zn}$ transporter (ZnT) 1-7, Zrt/Irt-like protein (Zip)-6, Zip-7 and solute carrier family 41 member 1 , which are involved in $\mathrm{Zn}$ transport, grow th hormone $(\mathrm{Gh})$, nuclear receptor subfamily 3 group $\mathrm{C}$ member 1 ( $\mathrm{Nr} 3 \mathrm{c} 1$, glucocorticoid receptor), ATP binding cassette subfamily $\mathrm{C}$ member 8 (Abcc8) and neuropeptide $\mathrm{Y}$ (Npy), which are involved in the regulation of lipid and energy metabolism, as well as glutamate ionotropic receptor AMPA type subunit 1 (GluR1) and 2 (GluR2), PRG-3, annexin A7 (ANX7), ANX3 and Bcl-2, which are involved in the regulation of neuroexcitability and neuroprotection. Of the twenty genes investigated, seven (ZnT-1, ZnT-2, Gh, Nr3c1, Abcc8, Npy and Bcl-2) were significantly downregulated, while four (ZnT-3, Zip-6, Zip-7 and PRG-3) were significantly upregulated in the RS group compared with CONT rats, which was only observed in the hippocampus (Figs. 2-4). No significant 


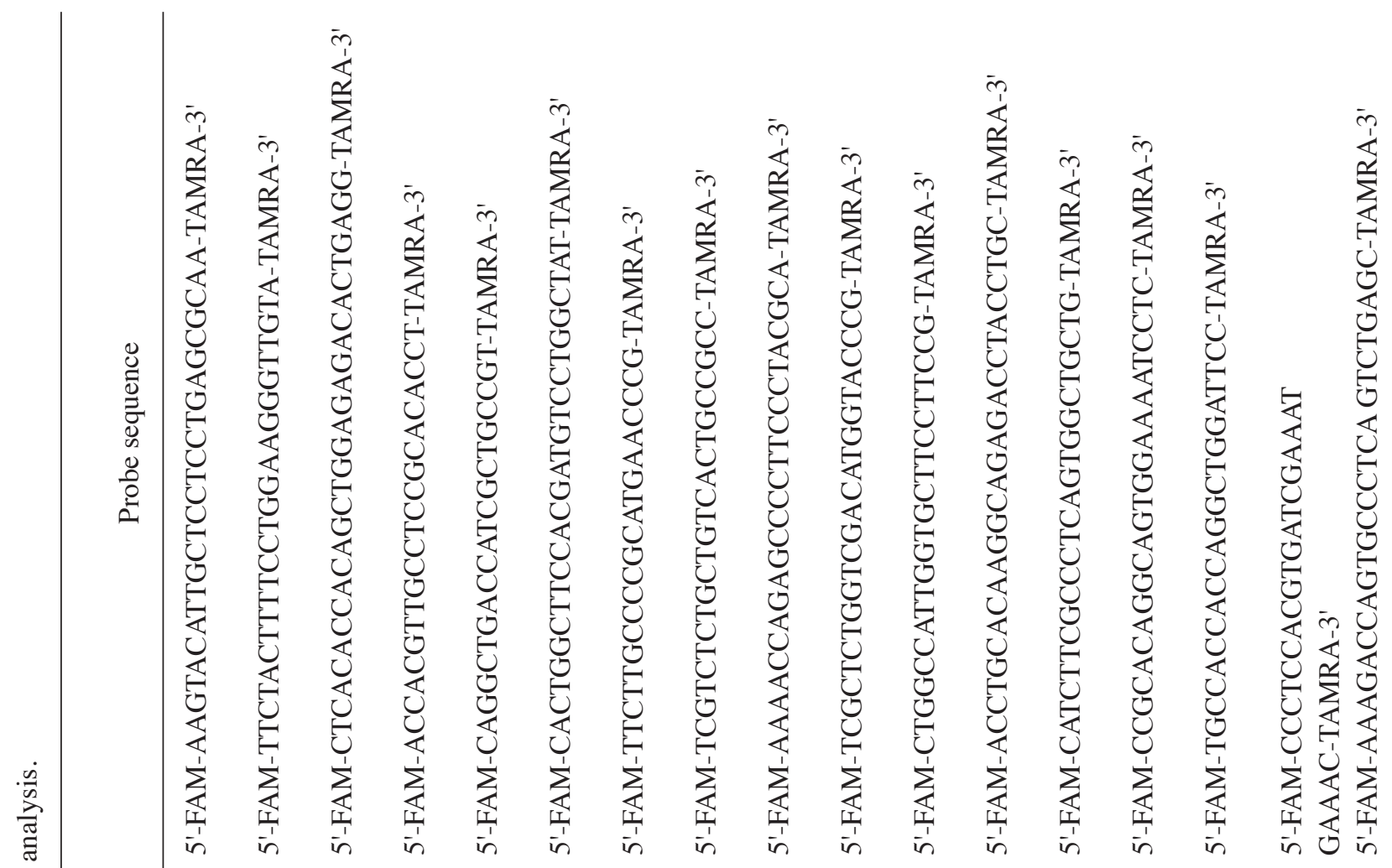

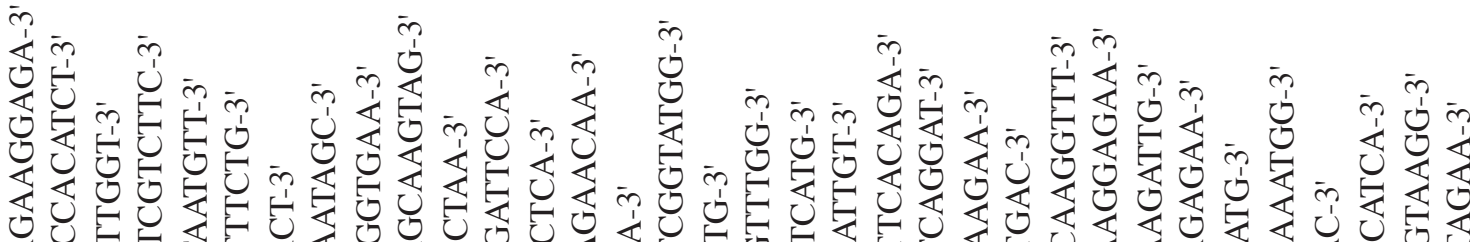

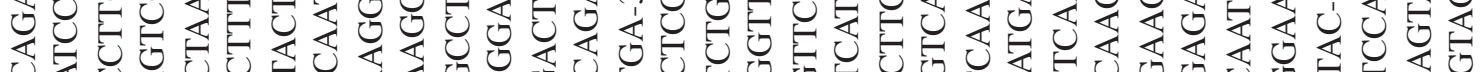

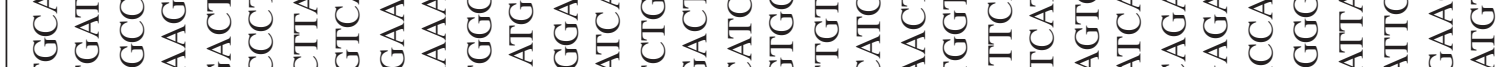

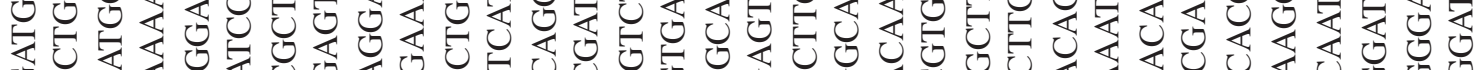

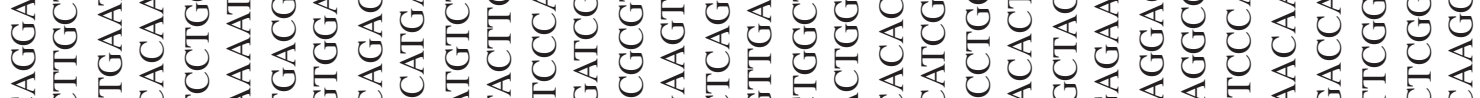

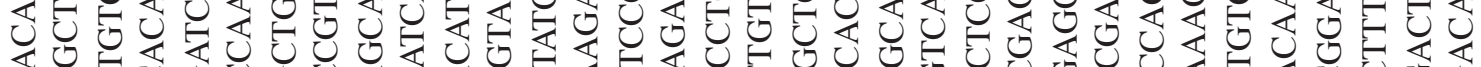

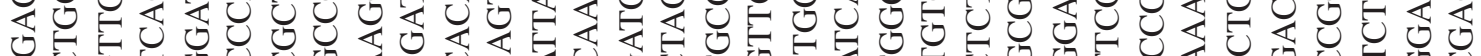

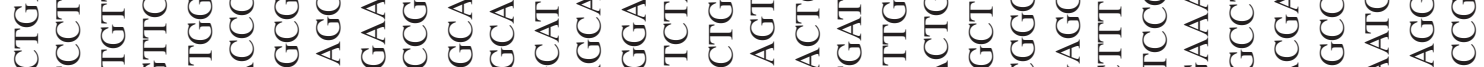

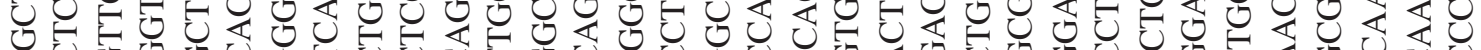

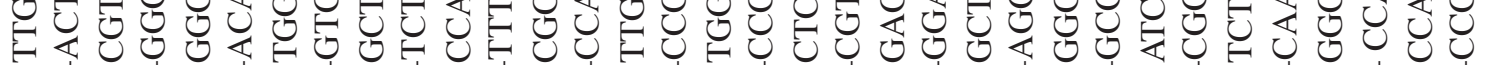
in in in in in in in in in in in in in in in in in in in in in in is in in in is is is in

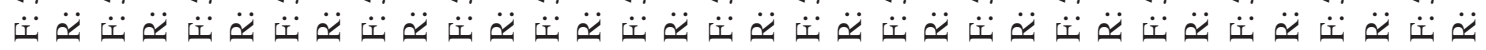

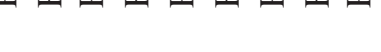


differences in gene expression were observed in the cerebral cortex between the two groups.

Linear correlation analysis among the eleven genes with significantly altered expression patterns (ZnT-1, ZnT-2, Gh, Nr3c1, Abcc8, Npy, Bcl-2, ZnT-3, Zip-6, Zip-7 and PRG-3) demonstrated that twenty-two different gene pairs exhibited significant positive or negative correlations, which accounted for $40 \%$ of the total of fifty-five gene pair combinations in the hippocampus (Fig. 5). The gene pairs that exhibited significant correlations in expression are as follows: Five pairs of genes among $\mathrm{Zn}$ transporters $\left(\mathrm{r}_{\mathrm{ZnT}-1 / \mathrm{ZnT}-2}=0.59\right.$, $\mathrm{P}<0.05 ; \mathrm{r}_{\text {ZnT-3/ZnT- } 2}=-0.62, \mathrm{P}<0.05 ; \mathrm{r}_{\text {Zip-6/ZnT }-3}=0.96, \mathrm{P}<0.0001$; $\left.\mathrm{r}_{\text {Zip-7/ZnT-3 }}=0.9, \mathrm{P}<0.0001 ; \mathrm{r}_{\text {Zip-7/Zip- } 6}=0.93, \mathrm{P}<0.0001\right)$; eight pairs of genes among metabolism-associated genes $\left(\mathrm{r}_{\mathrm{Npy} / \mathrm{Abcc} 8}=0.74, \mathrm{P}<0.001 ; \mathrm{r}_{\mathrm{Bcl}-2 / \mathrm{Gh}}=0.81, \mathrm{P}<0.01 ; \mathrm{r}_{\mathrm{Npy} / \mathrm{Gh}}=0.63\right.$, $\mathrm{P}<0.05 ; \mathrm{r}_{\mathrm{Nr} 3 \mathrm{cl} / \mathrm{Gh}}=0.85, \mathrm{P}<0.001 ; \mathrm{r}_{\mathrm{Bcl}-2 / \mathrm{PRG}-3}=-0.86, \mathrm{P}<0.001$; $\mathrm{r}_{\mathrm{PRG}-3 / \mathrm{Gh}}=-0.596, \mathrm{P}<0.05 ; \mathrm{r}_{\mathrm{Bcl}-2 / \mathrm{Npy}}=0.64, \mathrm{P}<0.05 ; \mathrm{r}_{\mathrm{PRG}-3 / \mathrm{Npy}}=-0.69$, $\mathrm{P}<0.05$ ); and nine pairs of genes between $\mathrm{Zn}$ transporter and metabolism-associated genes $\left(\mathrm{r}_{\mathrm{ZnT}-1 / \mathrm{Gh}}=0.58, \mathrm{P}<0.05\right.$; $\mathrm{r}_{\mathrm{ZnT}-2 / \mathrm{Gh}}=0.89, \mathrm{P}<0.001 ; \mathrm{r}_{\mathrm{ZnT}-3 / \mathrm{Gh}}=-0.67, \mathrm{P}<0.05 ; \mathrm{r}_{\mathrm{Bcl}-2 / \mathrm{ZnT} \mathrm{I}-\mathrm{l}} 0.75$,

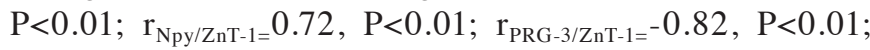
$\left.\mathrm{r}_{\mathrm{Nr} 3 \mathrm{c} / \mathrm{ZnT}-22}=0.69, \mathrm{P}<0.05 ; \mathrm{r}_{\mathrm{PRG}-3 / \mathrm{ZnT}-2=}=0.75, \mathrm{P}<0.01\right)$. Notably, the $\mathrm{r}$ values of twelve gene pairs in the hippocampus were $>0.7$ with $\mathrm{P}<0.01$. Of these twelve pairs, eight pairs were between $\mathrm{Zn}$ transporter and metabolism-associated genes or among $\mathrm{Zn}$ transporter genes (Fig. 5A), while four pairs were among metabolism-associated genes (Fig. 5B). Notably, Gh exhibited significant correlations with seven of the other genes that were analyzed (ZnT-1-ZnT-3, Nr3c1, Npy, PRG-3 and Bcl-2.). In addition, Gh also exhibited correlations with another three genes $\left(\mathrm{r}_{\mathrm{Zip} 6 / \mathrm{Gh}}=0.56, \mathrm{P}=0.056 ; \mathrm{r}_{\mathrm{Zip} 7 / \mathrm{Gh}=}=0.55, \mathrm{P}=0.064 ; \mathrm{r}_{\mathrm{Abcc8} / \mathrm{Gh}=} 0.56\right.$, $\mathrm{P}=0.06$ ), although these were not significant. Specifically, PRG-3 was significantly negatively correlated with Gh, Npy, Bcl-2, ZnT-1 and ZnT-2. However, only eight pairs of genes in the cerebral cortex exhibited significant positive correlations, which accounted for $14.5 \%$ of the total fifty-five gene pair combinations (Fig. 6).

\section{Discussion}

The present study demonstrated that flurothyl-treated rats exhibited aberrant mossy fiber sprouting in the hippocampus, as well as downregulated ZnT-1 and upregulated ZnT-3 expression. These results are consistent with our previous studies using flurothyl or penicillin-induced developmental seizure rat models $(9,10)$. Unlike our previous work, however, the results of the present study also indicated downregulated ZnT-2 and upregulated Zip-6/7 expression in the hippocampus. It has been previously reported that ZnT-1 and ZnT-3 are involved in $\mathrm{Zn}$ transport out of or into synaptic vesicles in the mossy fiber boutons, respectively, while ZnT-2 may prevent Zn causing toxicity in neurons by facilitating the movement of $\mathrm{Zn}$ into endosomes and lysosomes $(18,19)$. In addition, Zip proteins are involved in the transport of $\mathrm{Zn}$ from the extracellular space into the cytoplasm. Although the expression of Zip transporters (Zip-6 and Zip-7) has been reported in the brain $(20,21)$, to the best of our knowledge, their expression following developmental seizures has not previously been investigated. Therefore, the present study demonstrated for the first time that the expression of Zip-6/7 was altered at 
A

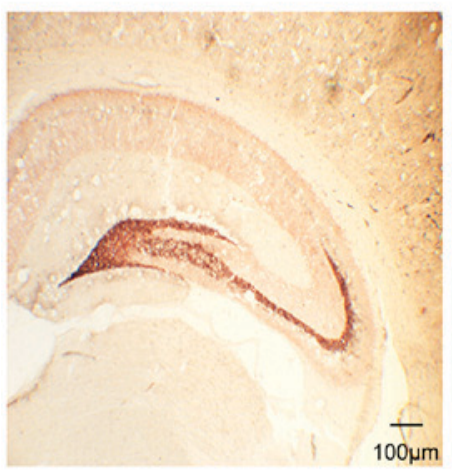

B

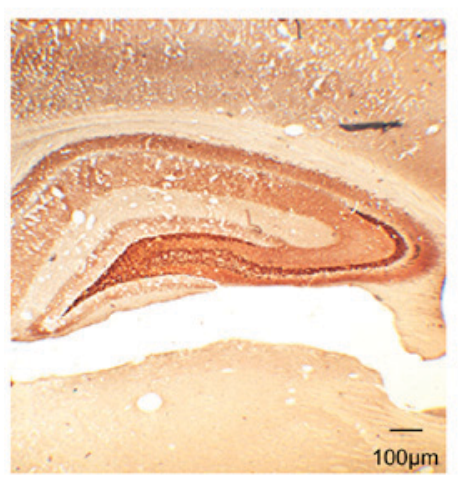

Dentate gyrus

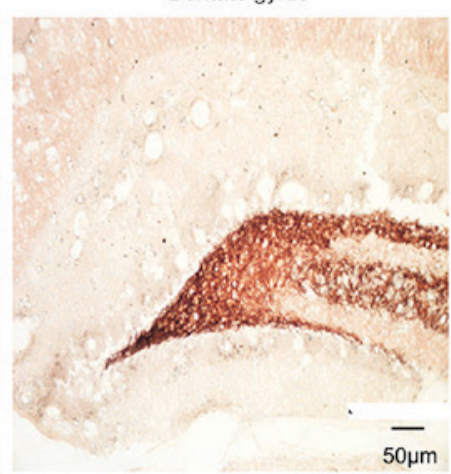

Dentate gyrus

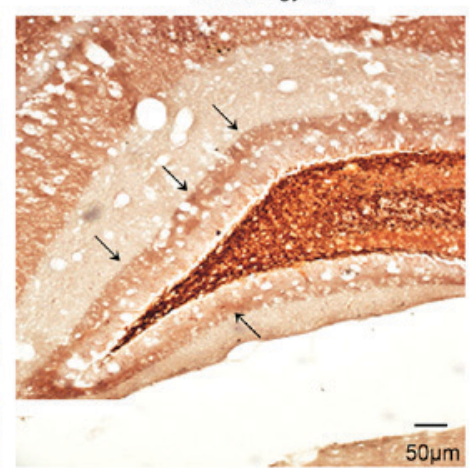

CA3

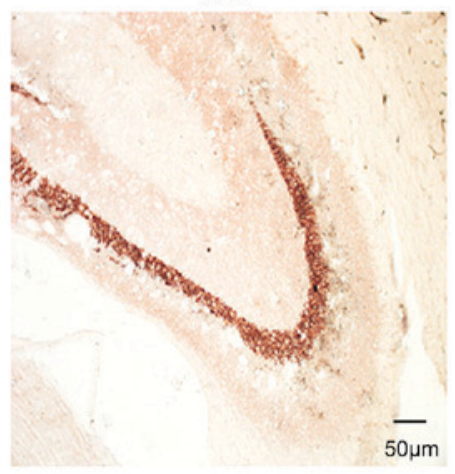

CA3

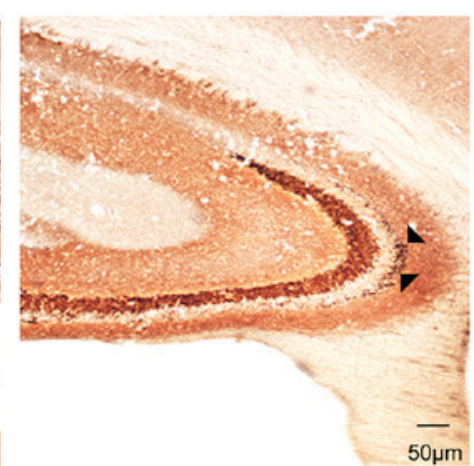

Figure 1. Mossy fiber sprouting by Timm staining in (A) CONT and (B) RS groups. Timm staining was used to investigate mossy fiber sprouting in the dentate gyrus and CA3 subfield of the hippocampus of rats in CONT and RS groups. Notably, excessive Timm staining was observed in the inner molecular layer of the granule cells in the dentate gyrus (arrow) and the stratum pyramidale of the CA3 subfield (arrowhead) in the RS group. CONT, control; RS, recurrent seizures; CA3, Cornu Ammonis 3.
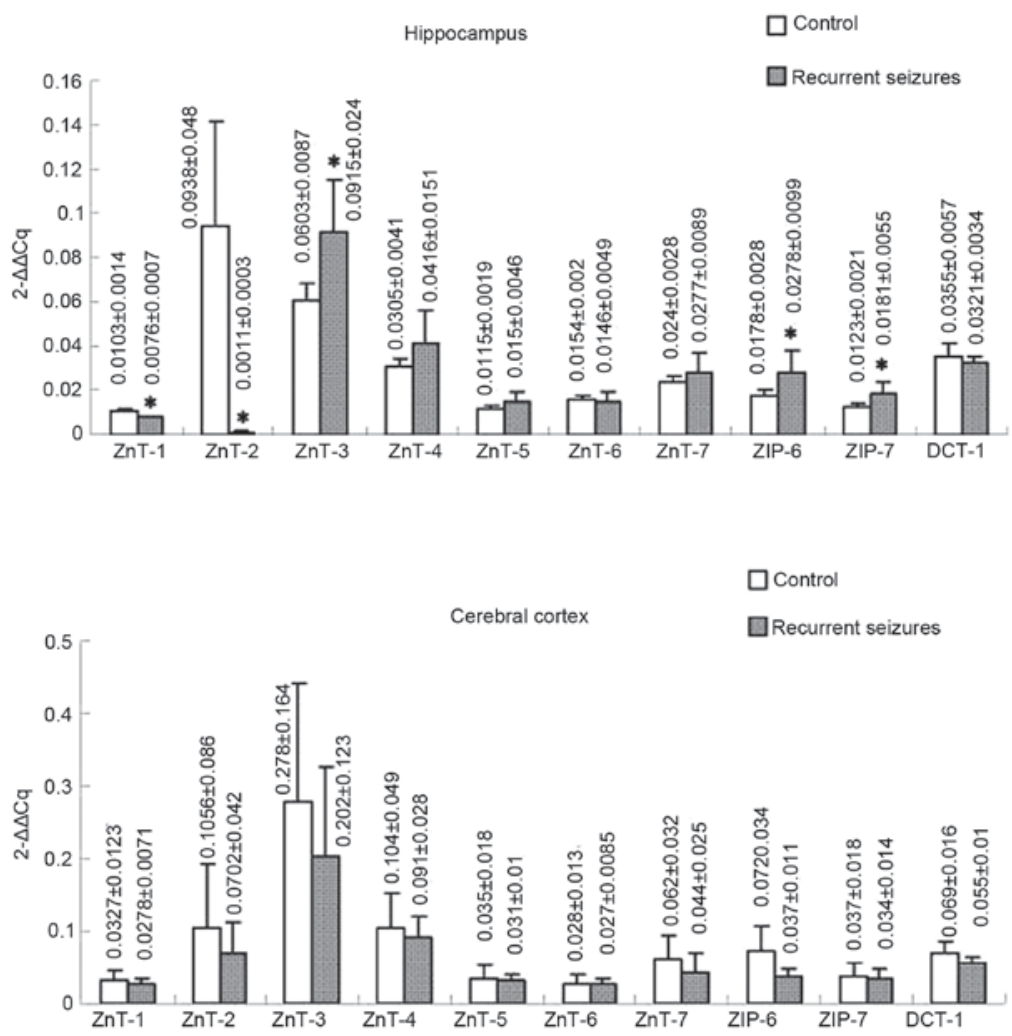

Figure 2. Reverse transcription-quantitative polymerase chain reaction analysis of $\mathrm{Zn}$ transporter-associated genes. When comparing mRNA levels between control and recurrent seizure groups, ZnT-1, ZnT-2, ZnT-3, Zip-6 and Zip-7 exhibited significant differences in the hippocampus. "P<0.05 vs. control. Zn, zinc; ZnT, Zn transporter; Zip, Zrt/Irt-like protein; DCT-1, solute carrier family 41 member 1. 

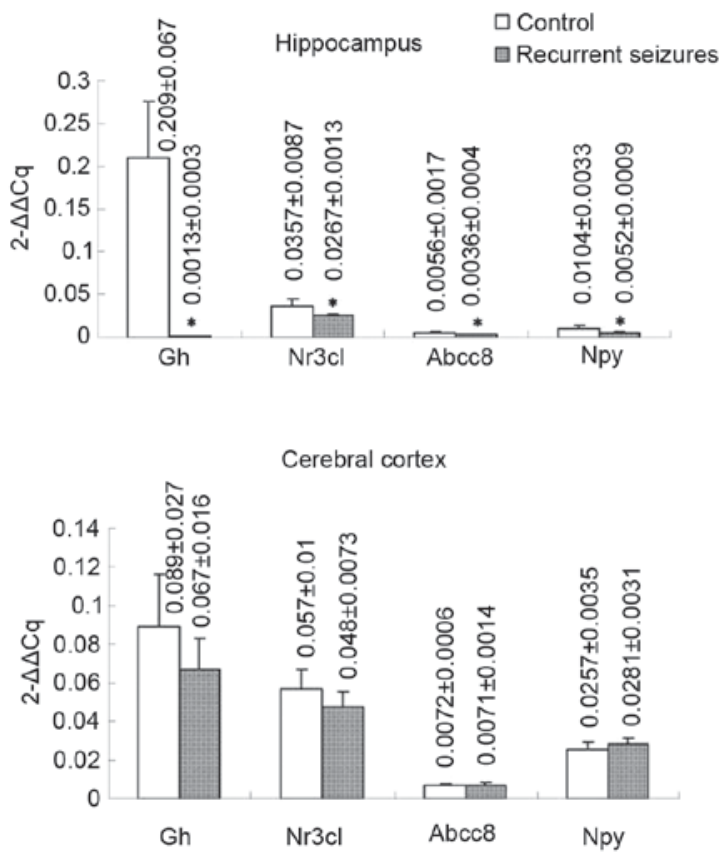

Figure 3. Reverse transcription-quantitative polymerase chain reaction analysis of lipid and energy metabolism-associated genes. When comparing mRNA levels between control and recurrent seizure groups, $\mathrm{Gh}, \mathrm{Nr} 3 \mathrm{cl}$, Abcc8 and Npy exhibited significant differences in the hippocampus. ${ }^{*} \mathrm{P}<0.05$ vs. control. Gh, growth hormone; $\mathrm{Nr} 3 \mathrm{c} 1$, nuclear receptor subfamily 3 group C member 1; Abcc8, ATP binding cassette subfamily C member 8; Npy, neuropeptide Y.

the transcriptional level in the hippocampus at a long-term time point following neonatal seizures, which may also be implicated in the intracellular $\mathrm{Zn}$ dyshomeostasis and pathophysiology of mossy fiber sprouting in the hippocampus.

Furthermore, the present study reported downregulated expressions of four lipid metabolism-associated genes. It is established that $\mathrm{Gh}$ is involved in the regulation of lipid metabolism (22). Disturbed expression of Gh in the hippocampus was reported to be associated with epilepsy progression in a model of TLE (23). Npy, an orexigenic hormone, is also involved in energy and lipid regulation and may act as a neuroprotective agent in the hippocampus during epileptic activities $(24,25)$. Cortisol binding to glucocorticoid receptors ( $\mathrm{Nr3c1}$ ) modulates lipid metabolism via glucocorticoid regulation. Glucocorticoids in the hippocampus have been reported to exhibit important roles in neuronal plasticity, electrical activity and cognitive functions (26). Abcc8 is one of the subunits of the ATP-sensitive potassium $\left(\mathrm{K}_{\mathrm{ATP}}\right)$ channel that connects energy metabolism to electrical excitability. It has recently been reported that $\mathrm{Nr} 3 \mathrm{c} 1$ and Abcc 8 mRNA levels in the hippocampus were significantly downregulated at 10 days or 2 months post status epilepticus in adult rats in the pilocarpine model of TLE (27). Consistent with these previous studies, the present results demonstrated decreased mRNA levels of the four lipid metabolism-associated genes at 21 days post the last neonatal seizure attacks. These results indicate that lipid metabolism-associated molecules, in addition to $\mathrm{Zn}$ transporters, may be associated with pathological changes in the hippocampus following developmental seizures. Although there is no direct evidence regarding the interaction between $\mathrm{Zn}$ and lipid metabolism pathways during epileptogenesis, recent progress concerning PRGs may provide valuable insight.
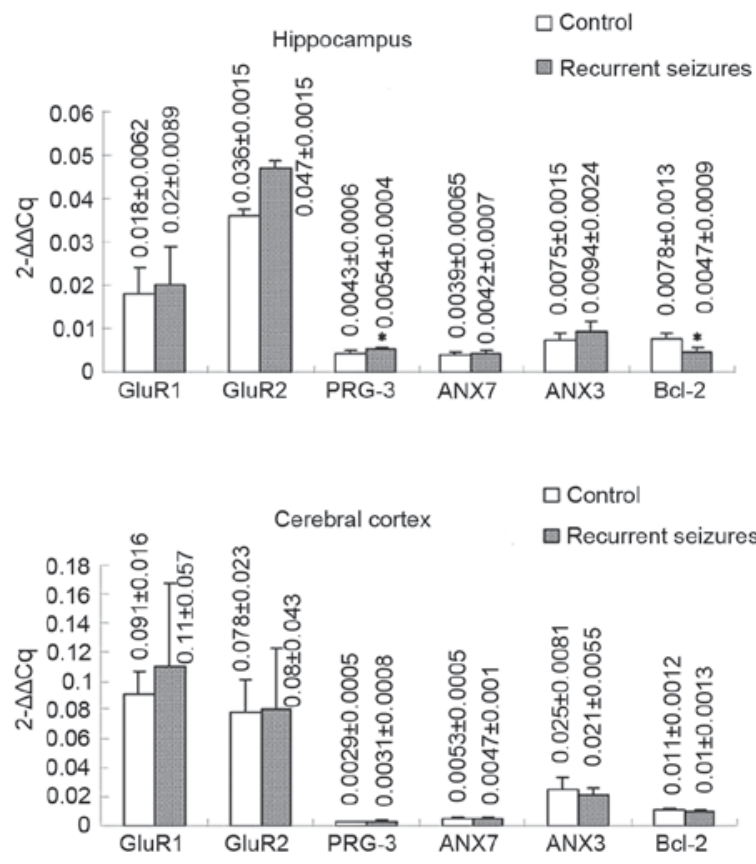

Figure 4. Reverse transcription-quantitative polymerase chain reaction analysis of neuroexcitability and neuroprotection-associated genes. When comparing mRNA levels between control and recurrent seizure groups, PRG-3 and Bcl-2 exhibited significant differences in the hippocampus. ${ }^{*} \mathrm{P}<0.05$ vs. control. PRG, plasticity-related gene; GluR1, glutamate ionotropic receptor AMPA type subunit 1; GluR2, glutamate ionotropic receptor AMPA type subunit 2; ANX7, annexin A7; ANX3, annexin A3.

PRGs are a subclass of the lipid phosphate phosphatase (LPP) superfamily. Unlike traditional LPPs, PRGs are predominantly expressed in the central nervous system, and may modify bioactive lipids and the associated signaling pathways to exert their function (28). For example, PRG-1 functions as an ectoenzyme and was reported to prevent phospholipid-induced axon collapse in neurons and enhance hippocampal outgrowth (29,30). Previously, using a semi-quantitative RT-PCR method, we investigated the dynamic pattern of the gene expression of ZnT-1, ZnT-3 and PRG-1 in the rat brain following flurothyl-induced prolonged neonatal seizures at $1.5,3,6,12,24$ and $48 \mathrm{~h}$, and 7 and 14 days after the last induced seizure. The results demonstrated that long-term, 14 days after the last seizure, ZnT-3 mRNA and PRG-1 protein levels in the hippocampus were upregulated, while downregulated mRNA expression of ZnT-1 was observed (11). These results are consistent with the results of the current study. The present study also reported a similar decrease in ZnT-1, and upregulated ZnT-3 and PRG-3 expression in the hippocampus at 21 days after the last seizure, and sprouting of hippocampal mossy fibers was also observed. Linear correlation analysis further demonstrated that PRG-3 was significantly negatively correlated with Gh, Npy, Bcl-2, ZnT-1 and ZnT-2. In addition, a further seven pairs of genes between $\mathrm{Zn}$ transporter and lipid metabolism-associated molecules exhibited significant positive correlations. These results may support the results of a study by Blanco-Alvarez et al (31), which reported an increase of $\mathrm{Zn}$ and lipoperoxidation levels, cell death by necrosis and apoptosis in the late phase of ischemia in the rat temporoparietal cortex and hippocampus when pretreated with $\mathrm{Zn}$ and receiving L-NAME administration $1 \mathrm{~h}$ prior to common 

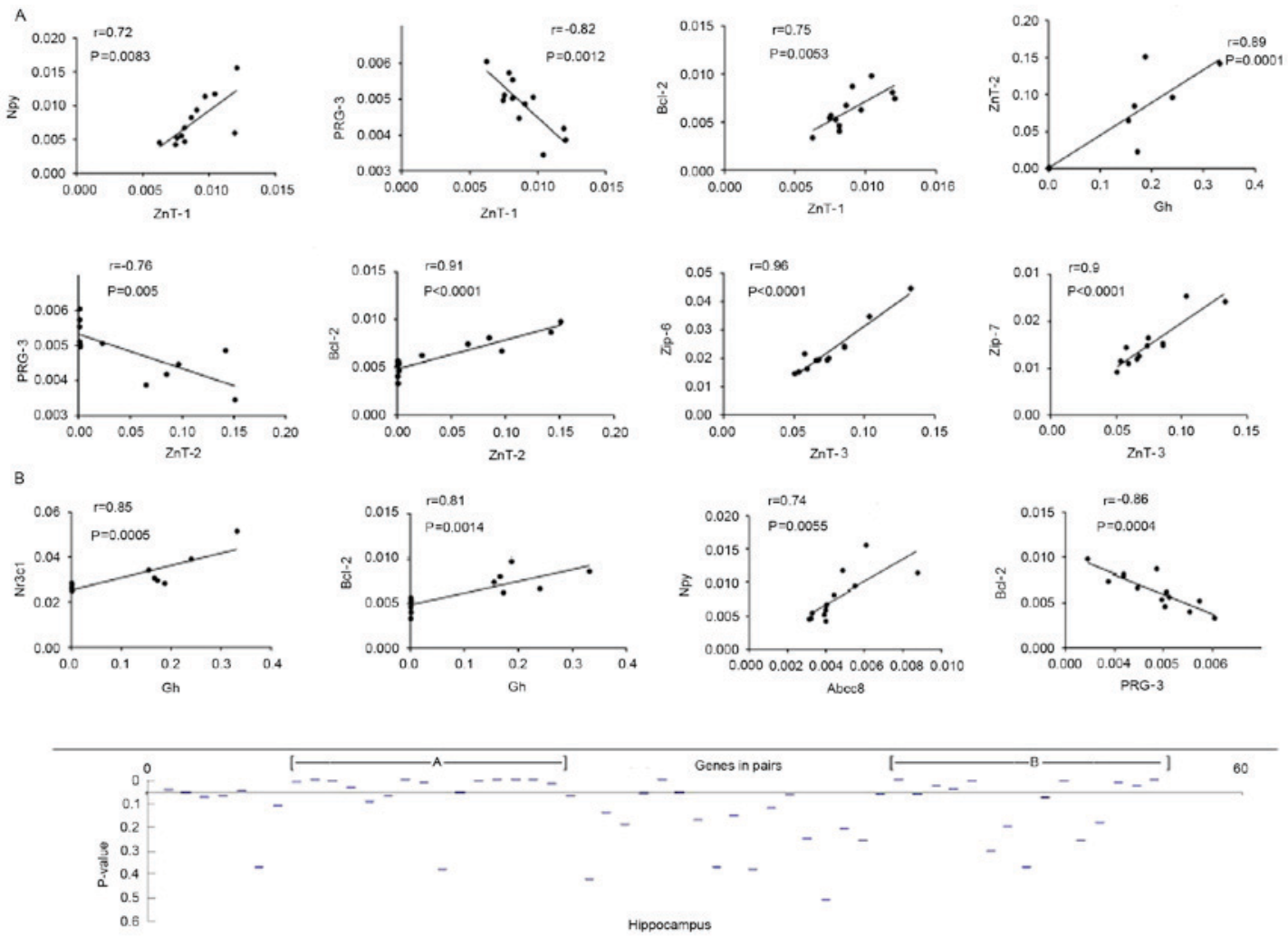

Figure 5. Linear correlation analysis of reverse transcription-quantitative polymerase chain reaction results in the hippocampus. (A) The r-values of eight gene pairs were $>0.7$ with $\mathrm{P}<0.01$, and the majority of these gene pairs consisted of a zinc transporter and a metabolism-associated gene. (B) Upper, the r-values of four gene pairs were $>0.7$ with $\mathrm{P}<0.01$, with pairs consisting of metabolism-associated genes and neuroexcitability/neuroprotection-associated genes. Lower, significant positive correlations $(\mathrm{P}<0.05)$ were observed for twenty-two gene pairs, which accounted for $40 \%$ of the total fifty-five gene pair combinations. Npy, neuropeptide Y; ZnT, zinc transporter; PRG, plasticity-related gene; Gh, growth hormone; Zip, Zrt/Irt-like protein; Nr3c1, nuclear receptor subfamily 3 group C member 1; Abcc8, ATP binding cassette subfamily C member 8.
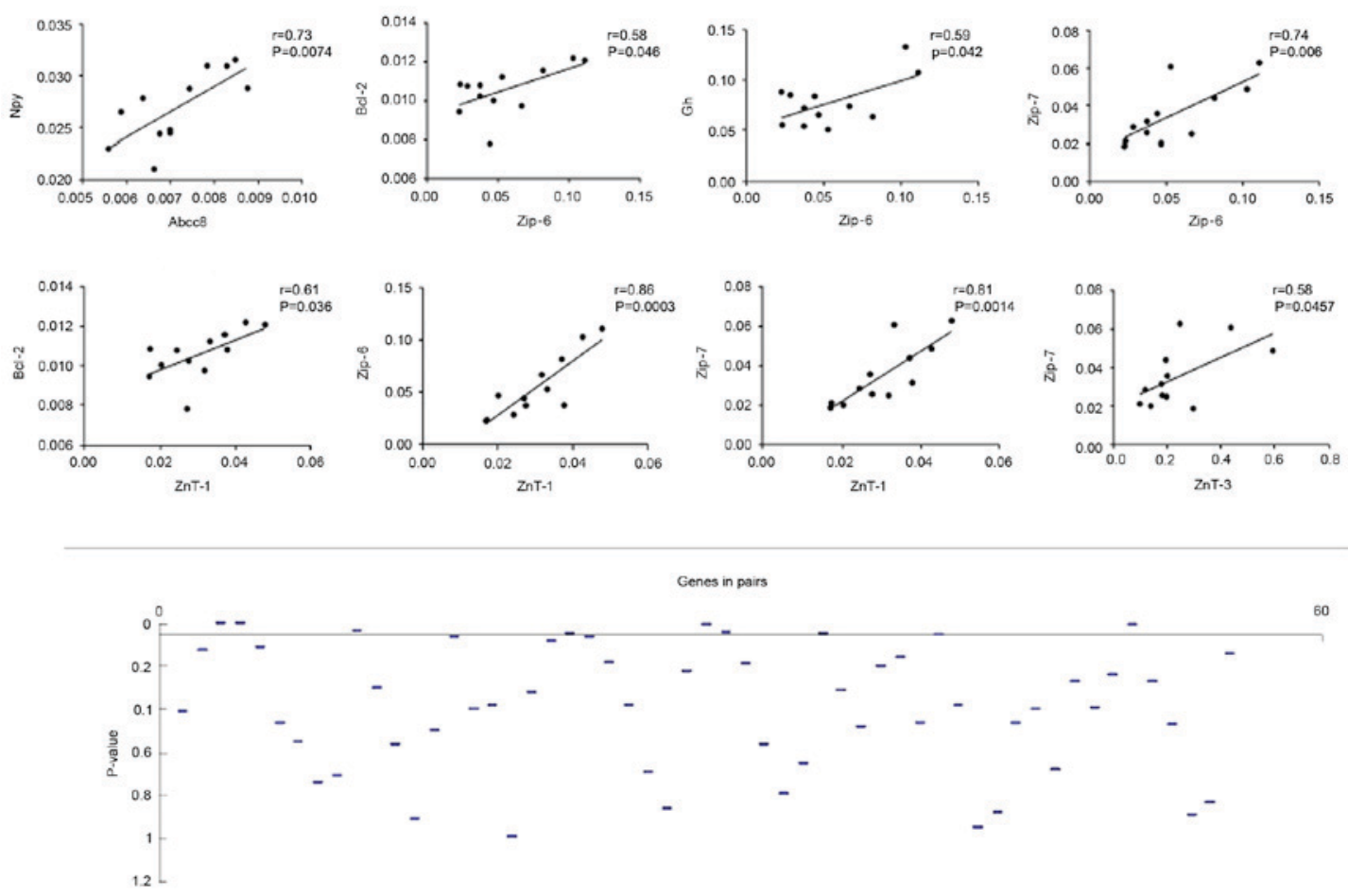

Cerebral corte:

Figure 6. Linear correlation analysis of reverse transcription-quantitative polymerase chain reaction results in the cerebral cortex. Only eight pairs of genes exhibited significant positive correlations, which accounted for $14.5 \%$ of the total fifty-five gene pair combinations. Npy, neuropeptide Y; Abcc8, ATP binding cassette subfamily C member 8; Zip, Zrt/Irt-like protein; Gh, growth hormone; ZnT, zinc transporter. 
carotid-artery occlusion, indicating a synergistic relationship between $\mathrm{Zn}$ and lipid metabolism signals during brain injury. Therefore, it may be hypothesized a synergistic interaction exists between $\mathrm{Zn}$ and lipid metabolism pathways during epileptogenesis, which requires further investigation.

In addition, the present study demonstrated a potential increase in hippocampal apoptosis as evidenced by the downregulated expression of $\mathrm{Bcl}-2$ in the hippocampus in flurothyl-treated rats. Bcl-2 is an apoptosis suppressor and it has been reported that downregulated Bcl-2 expression contributes to apoptotic cell death during acute brain damage following experimental seizures $(32,33)$. However, it is not clear whether developmental seizure attacks lead to long-term disturbance of apoptosis-associated signaling pathways during adulthood. In the current study, decreased mRNA levels of Bcl-2 in the hippocampus were observed at the long-term time point after seizures. Due to the lack of the results regarding other apoptosis-associated molecules, our observations only indicate a potential disrupted apoptosis-associated signal at the mRNA level. The long-term effects of developmental seizures on apoptosis in the hippocampus requires investigation in future experimental studies.

The current study has certain limitations. For example, the present study did not investigate the cell types in the hippocampus that contributed to the changes in gene expression. However, previous studies have demonstrated that Gh, Npy, PRG-3 and ZnT-3 are synthesized by hippocampal neurons, where their expression is affected by exposure to stress (34-40). The results of the current study demonstrated significantly up or downregulated expression of these four genes accompanied by strong correlations in expression following seizures. These results indicate that the cell type responsible for the observed results in the present study may be neurons. However, as immunohistochemistry or in situ hybridization studies were not performed in the present study, other cell types, including astrocytes or microglia, may also be involved in the expression of the regulated genes, which also requires further investigation.

In conclusion, the current study demonstrated, for the first time, based on molecular evidence, that altered expression of $\mathrm{Zn}$ transporter and metabolism-associated genes may be associated with neonatal seizure-induced aberrant mossy fiber sprouting in the hippocampus, and may be potential targets for the treatment of neonatal seizure-induced brain injury.

\section{Acknowledgements}

This work was supported by the National Natural Science Foundation of China (grant nos. 81271458 and 81471337) and Jiangsu Province's Key Provincial Talents Program (grant no. RC2011113).

\section{References}

1. Ben-Ari Y and Holmes GL: Effects of seizures on developmental processes in the immature brain. Lancet Neurol 5: 1055-1063, 2006.

2. Nardou R, Ferrari DC and Ben-Ari Y: Mechanisms and effects of seizures in the immature brain. Semin Fetal Neonatal Med 18: 175-184, 2013.

3. Sanchez RM and Jensen FE: Maturational aspects of epilepsy mechanisms and consequences for the immature brain. Epilepsia 42: 577-585, 2001.
4. Thom M: Review: Hippocampal sclerosis in epilepsy: A neuropathology review. Neuropathol Appl Neurobiol 40: 520-543, 2014.

5. Sayin U, Hutchinson E, Meyerand ME and Sutula T: Age-dependent long-term structural and functional effects of early-life seizures: Evidence for a hippocampal critical period influencing plasticity in adulthood. Neuroscience 288: 120-134, 2015.

6. Rakhade SN and Jensen FE: Epileptogenesis in the immature brain: Emerging mechanisms. Nat Rev Neurol 5: 380-391, 2009.

7. GrabruckerS,Jannetti L,EckertM,GaubS, Chhabra R,PfaenderS, Mangus K, Reddy PP, Rankovic V, Schmeisser MJ, et al: Zinc deficiency dysregulates the synaptic ProSAP/Shank scaffold and might contribute to autism spectrum disorders. Brain 137: 137-152, 2014.

8. Sanchez RM, Ribak CE and Shapiro LA: Synaptic connections of hilar basal dendrites of dentate granule cells in a neonatal hypoxia model of epilepsy. Epilepsia 53 (Suppl 1): S98-S108, 2012.

9. Ni H, Jiang YW, Tao LY, Jin MF and Wu XR: ZnT-1, ZnT-3, CaMKII, PRG-1 expressions in hippocampus following neonatal seizure-induced cognitive deficit in rats. Toxico Lett 184: 145-150, 2009.

10. Ni H, Jiang YW, Tao LY, Cen JN and Wu XR: Effects of penicillin-induced developmental epilepticus on hippocampal regenerative sprouting, related gene expression and cognitive deficits in rats. Toxico Lett 188: 161-166, 2009.

11. Ni H, Jiang YW, Xiao ZJ, Tao LY, Jin MF and Wu XR: Dynamic pattern of gene expression of ZnT-1, ZnT-3 and PRG-1 in rat brain following flurothyl-induced recurrent neonatal seizures. Toxicol Lett 194: 86-93, 2010.

12. Ni H, Ren SY, Zhang LL, Sun Q, Tian T and Feng X: Expression profiles of hippocampal regenerative sprouting-related genes and their regulation by E-64d in a developmental rat model of penicillin-induced recurrent epilepticus. Toxicol Lett 217: 162-169, 2013.

13. Tian T, Ni H and Sun BL: Neurobehavioral deficits in a rat model of recurrent neonatal seizures are prevented by a ketogenic diet and correlate with hippocampal Zinc/Lipid transporter signals. Biol Trace Elem Res 167: 251-258, 2015.

14. Scholl EA, Dudek FE and Ekstrand JJ: Neuronal degeneration is observed in multiple regions outside the hippocampus after lithium pilocarpine-induced status epilepticus in the immature rat. Neuroscience 252: 45-59, 2013.

15. Cepeda C, André VM, Levine MS, Salamon N, Miyata H, Vinters HV and Mathern GW: Epileptogenesis in pediatric cortical dysplasia: The dysmature cerebral developmental hypothesis. Epilepsy Behav 9: 219-235, 2006.

16. Ni H, Feng X, Gong Y, Tao LY and Wu XR: Acute phase expression pattern of ZnTs, LC3, and beclin-1 in rat Hippocampus and its regulation by 3 -methyladenine following recurrent neonatal seizures. Biol Trace Elem Res 143: 320-331, 2011.

17. Livak KJ and Schmittgen TD: Analysis of relative gene expression data using real-time quantitative PCR and the 2(-Delta Delta C(T)) method. Methods 25: 402-408, 2001.

18. Colvin RA, Bush AI, Volitakis I, Fontaine CP, Thomas D, Kikuchi $\mathrm{K}$ and Holmes WR: Insights into Zn2+ homeostasis in neurons from experimental and modeling studies. Am J Physiol Cell Physiol 294: C726-C742, 2008.

19. Murakami M and Hirano T: Intracellular zinc homeostasis and zinc signaling. Cancer Sci 99: 1515-1522, 2008.

20. Nakashima AS and Dyck RH: Zinc and cortical plasticity. Brain Res Rev 59: 347-373, 2009.

21. Szewczyk B: Zinc homeostasis and neurodegenerative disorders Front Aging Neurosci 5: 33, 2013.

22. Faylon MP, Koltes DE and Spurlock DM: Regulation of lipid droplet-associated proteins following growth hormone administration and feed restriction in lactating Holstein cows. J Dairy Sci 97: 2847-2855, 2014.

23. Kato K, Suzuki M, Kanno H, Sekino S, Kusakabe K, Okada T, Mori T, Yoshida K and Hirabayashi Y: Distinct role of growth hormone on epilepsy progression in a model of temporal lobe epilepsy. J Neurochem 110: 509-519, 2009.

24. Schwartz MW: Central nervous system regulation of food intake. Obesity (Silver Spring) 14 (Suppl 1): S1-S8, 2006.

25. Vezzani A, Sperk G and Colmers WF: Neuropeptide Y: Emerging evidence for a functional role in seizure modulation. Trends Neurosci 22: 25-30, 1999.

26. Snyder JS, Soumier A, Brewer M, Pickel J and Cameron HA: Adult hippocampal neurogenesis buffers stress responses and depressive behaviour. Nature 476: 458-461, 2011. 
27. Ruiz N, Pacheco LF, Farrell B, Cox CB, Ermolinsky BS, Garrido-Sanabria ER and Nair S: Metabolic gene expression changes in the hippocampus of obese epileptic male rats in the pilocarpine model of temporal lobe epilepsy. Brain Res 1426: 86-95, 2011.

28. Strauss U and Bräuer AU: Current views on regulation and function of plasticity-related genes (PRGs/LPPRs) in the brain. Biochim Biophys Acta 1831: 133-138, 2013.

29. Bräuer $A U$ and Nitsch R: Plasticity-related genes (PRGs/LRPs): A brain-specific class of lysophospholipid-modifying proteins. Biochim Biophys Acta 1781: 595-600, 2008.

30. Bräuer AU, Savaskan NE, Kühn H, Prehn S, Ninnemann O and Nitsch R: A new phospholipid phosphatase, PRG-1, is involved in axon growth and regenerative sprouting. Nat Neurosci 6: $572-578,2003$

31. Blanco-Alvarez VM, Lopez-Moreno P, Soto-Rodriguez G, Martinez-Fong D, Rubio H, Gonzalez-Barrios JA, Piña-Leyva C, Torres-Soto M, Gomez-Villalobos Mde J, Hernandez-Baltazar D, et al: Subacute zinc administration and L-NAME caused an increase of NO, zinc, lipoperoxidation, and caspase-3 during a cerebral hypoxia-ischemia process in the rat. Oxid Med Cell Longev 2013: 240560, 2013.

32. Sun J, Xie C, Liu W, Lu D, Qiao W, Huang Q, Huo Z, Shen H and Lin Z: The effects of simvastatin on hippocampal caspase-3 and Bcl-2 expression following kainate-induced seizures in rats. Int J Mol Med 30: 739-746, 2012.

33. Henshall DC and Engel T: Contribution of apoptosis-associated signaling pathways to epileptogenesis: Lessons from Bcl-2 family knockouts. Front Cell Neurosci 7: 110, 2013.
34. Vander Weele CM, Saenz C, Yao J, Correia SS and Goosens KA: Restoration of hippocampal growth hormone reverses stress-induced hippocampal impairment. Front Behav Neurosci 7: 66, 2013.

35. Donahue CP, Jensen RV, Ochiishi T, Eisenstein I, Zhao M, Shors T and Kosik KS: Transcriptional profiling reveals regulated genes in the hippocampus during memory formation. Hippocampus 12: 821-833, 2002.

36. Goto EM, Silva Mde P, Perosa SR, Argañaraz GA, Pesquero JB, Cavalheiro EA, Naffah-Mazzacoratti MG, Teixeira VP and Silva JA Jr: Akt pathway activation and increased neuropeptide Y mRNA expression in the rat hippocampus: Implications for seizure blockade. Neuropeptides 44: 169-176, 2010.

37. Drexel M, Kirchmair E, Wieselthaler-Hölzl A, Preidt AP and Sperk G: Somatostatin and neuropeptide Y neurons undergo different plasticity in parahippocampal regions in kainic acid-induced epilepsy. J Neuropathol Exp Neurol 71: 312-329, 2012.

38. Savaskan NE, Bräuer AU and Nitsch R: Molecular cloning and expression regulation of PRG-3, a new member of the plasticity-related gene family. Eur J Neurosci 19: 212-220, 2004.

39. Velmans T, Battefeld A, Geist B, Farrés AS, Strauss U and Bräuer AU: Plasticity-related gene 3 promotes neurite shaft protrusion. BMC Neurosci 14: 36, 2013.

40. Chi ZH, Wang X, Cai JQ, Stoltenberg M, Danscher G and Wang ZY: Zinc transporter 3 immunohistochemical tracing of sprouting mossy fibres. Neurochem Int 52: 1305-1309, 2008. 